ARTICLE

\title{
Two-dimensional $\mathrm{Mo}_{1.33} \mathrm{C}$ MXene with divacancy ordering prepared from parent 3D laminate with in-plane chemical ordering
}

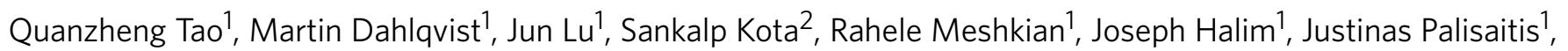
Lars Hultman ${ }^{1}$, Michel W. Barsoum ${ }^{1,2}$, Per O.A. Persson ${ }^{1} \&$ Johanna Rosen ${ }^{1}$

The exploration of two-dimensional solids is an active area of materials discovery. Research in this area has given us structures spanning graphene to dichalcogenides, and more recently 2D transition metal carbides (MXenes). One of the challenges now is to master ordering within the atomic sheets. Herein, we present a top-down, high-yield, facile route for the controlled introduction of ordered divacancies in MXenes. By designing a parent $3 \mathrm{D}$ atomic laminate, $\left(\mathrm{Mo}_{2 / 3} \mathrm{Sc}_{1 / 3}\right)_{2} \mathrm{AIC}$, with in-plane chemical ordering, and by selectively etching the $\mathrm{Al}$ and $\mathrm{SC}$ atoms, we show evidence for $2 \mathrm{D} \mathrm{Mo}_{1.33} \mathrm{C}$ sheets with ordered metal divacancies and high electrical conductivities. At $\sim 1,100 \mathrm{Fcm}^{-3}$, this $2 \mathrm{D}$ material exhibits a $65 \%$ higher volumetric capacitance than its counterpart, $\mathrm{Mo}_{2} \mathrm{C}$, with no vacancies, and one of the highest volumetric capacitance values ever reported, to the best of our knowledge. This structural design on the atomic scale may alter and expand the concept of property-tailoring of 2D materials.

\footnotetext{
${ }^{1}$ Thin Film Physics, Department of Physics, Chemistry and Biology (IFM), Linköping University, SE-581 83 Linköping, Sweden. ${ }^{2}$ Department of Materials Science and Engineering, Drexel University, Philadelphia, Pennsylvania 19104, USA. Correspondence and requests for materials should be addressed to J.R. (email: johanna.rosen@liu.se).
} 
T wo-dimensional (2D) ultrathin materials, for example, graphene, $\mathrm{BN}$ and $\mathrm{MoS}_{2}$, possess unique properties, which, can be utilized for various applications ${ }^{1-3}$. Defects, including vacancies, in these materials have been extensively studied, and shown to influence magnetic, electronic, catalytic and optoelectronic characteristics ${ }^{4-8}$. However, these defects are typically either unintentional or formed in macroscopic processes where the defect type and/or extent are not highly controlled. The prevailing challenge therefore is to master the order within the atomic sheets, on an atomic level, at a large scale.

One of the more promising applications of $2 \mathrm{D}$ solids is in the realm of energy storage, where intrinsically high specific surface areas and fast ionic diffusion through $2 \mathrm{D}$ channels result in high energy and power density electrodes. For example, previous reports on free-standing graphene-based electrodes ${ }^{9-11}$ demonstrate that combining large specific surface areas with good electrical conductivity has led to specific capacitances of up to $300 \mathrm{~F} \mathrm{~cm}^{-3}$. When electrically conductive $2 \mathrm{D}$ materials are capable of pseudocapacitive charge storage (that is, fast surface redox reactions) ${ }^{12}$, significantly higher energy and power densities can be achieved, as in the case of $\mathrm{RuO}_{2}$ (ref. 13) or $\mathrm{Ti}_{3} \mathrm{C}_{2}$ MXene ${ }^{14}$.

Discovered in 2011, MXenes are a comparatively young class of $2 \mathrm{D}$ materials obtained by the selective etching of A-group elements, mostly $\mathrm{Al}$, from atomically laminated parent 3D $\mathrm{M}_{n+1} \mathrm{AX}_{n}$ (MAX) phases (where $\mathrm{M}$ is a transition metal, $\mathrm{A}$ is an A-group element, and $\mathrm{X}$ is $\mathrm{C}$ and/or $\mathrm{N})^{15}$. When the A-element is etched, it is replaced by surface terminations, $\mathrm{T}_{x}$, and hence the proper MXene formula is $\mathrm{M}_{n+1} \mathrm{X}_{n} \mathrm{~T}_{x}$, where $\mathrm{T}_{x}$ is a combination of $-\mathrm{O},-\mathrm{OH}$ and $-\mathrm{F}^{16}$. In many applications from energy storage ${ }^{14,17,18}$ to cationic adsorption ${ }^{19}$, conductive transparent electrodes ${ }^{20}$, and electromagnetic interference shielding ${ }^{21}$, MXenes perform as well as, if not better than, anything before them.

While it has long been appreciated that solid solutions can be formed on the M-, A- or X-sites, it is only quite recently that a quaternary MAX phase, $\mathrm{Cr}_{2} \mathrm{TiAlC}_{2}$, with out-of-plane chemical ordering was reported ${ }^{22}$. Several other out-of-plane MAX phases have since been discovered, and crucially the out-of-plane structure is preserved in their corresponding MXenes ${ }^{23,24}$. Herein we not only report on a type of ordered quaternary $\left(\mathrm{M}_{2 / 3}^{1} \mathrm{M}_{1 / 3}^{2}\right)_{2} \mathrm{AlC}$ phase that we coin ' $\mathrm{i}$-MAX', wherein $\mathrm{M}^{1}$ and $\mathrm{M}^{2}$ are ordered inplane, but also show how etching such solids results in $2 \mathrm{D}$ materials with ordered divacancies. A schematic of the MAX to MXene transformation is shown in Fig. 1a, where the divacancy configuration is best seen in the background schematic in Fig. 1a (right panel). We also report that this 2D material exhibits one of the highest conductivities of any MXene and possesses one of the highest specific capacitances, to the best of our knowledge. This introduction of ordered divacancies represents a breakthrough for property tuning of $2 \mathrm{D}$ materials which, critically, is also accompanied by a reduction in mass density.

\section{Results}

In-plane chemical ordering in a 3D MAX phase. The quaternary $\mathrm{MAX}\left(\mathrm{Mo}_{2 / 3} \mathrm{Sc}_{1 / 3}\right)_{2} \mathrm{AlC}$ with in-plane ordering, left panel in Fig. 1a, was discovered through the interplay between material synthesis and characterization followed by first-principles calculations based on Density Functional Theory (DFT) (see Methods section below). The calculations show that this phase is the most stable with respect to all competing phases in the Mo-Sc-Al-C system (Supplementary Table 1), and that the monoclinic $(\mathrm{C} 2 / \mathrm{c})$ and orthorhombic $(\mathrm{Cmcm})$ structures are almost degenerate in energy (Supplementary Fig. 1 and Supplementary Table 2). Furthermore, from the calculated phonon dispersion spectra (Supplementary Fig. 2) we conclude that $\left(\mathrm{Mo}_{2 / 3} \mathrm{Sc}_{1 / 3}\right)_{2} \mathrm{AlC}$ is also dynamically stable, that is, stable to lattice vibrations with no imaginary phonon frequencies.

Scanning transmission electron microscopy (STEM) images of the MAX phase along [010], [110] and [100] zone axes are shown, respectively, in Fig. 1b-d. In these micrographs, the Mo atoms appear brightest; the $\mathrm{Sc}$ and $\mathrm{Al}$ atoms are less bright. The schematics to the left of each micrograph sketch the atomic arrangements expected, assuming monoclinic $\left(\mathrm{Mo}_{2 / 3} \mathrm{Sc}_{1 / 3}\right)_{2} \mathrm{AlC}$. In Fig. 1d, the structure looks identical to a traditional MAX phase viewed along the [11-20] zone axis. However, evidence for in-plane ordering of the two transition metals is revealed in Fig. 1b,c, respectively, where the former image rules out orthorhombic symmetry (Supplementary Fig. 1). Most notably, the Sc atoms extend out of the Mo planes towards the $\mathrm{Al}$ layers. Consequently, the Al layers form a Kagomé lattice, evident from the bright-dark contrast variation in that layer. Lastly, the in-plane ordered structure is also revealed by the selective area electron diffraction shown in the insets of Fig. 1b-d.

Figure 1e shows the XRD patterns of a sample with nominal composition $\left(\mathrm{Mo}_{2 / 3} \mathrm{Sc}_{1 / 3}\right)_{2} \mathrm{AlC}$, for which the metal ratios are confirmed by energy-dispersive X-ray analysis (EDX). The Mo/Sc atomic ratio was found to be close to two (Supplementary Fig. 3). The Rietveld refinement based on space group C2/c (\#15) is included in Fig. 1e, with the refinement parameters listed in Supplementary Table 3. Note that while the material before etching is not single phase, after etching it is (see below).

The lattice parameters calculated from the XRD Rietveld analysis, $a=9.3486(1) \AA, b=5.3985(1) \AA$ and $c=13.8738(2) \AA$, are consistent with theory and closer to those for the monoclinic than the orthorhombic structure (Supplementary Table 2). Note that the 110 peak-corresponding to an interplanar distance of $4.7 \AA$-appears in the XRD pattern around $19^{\circ}$ (inset in Fig. 1e). This peak does not exist in the ternary 211 phases. This superstructure peak thus occurs solely due to $\mathrm{Mo} / \mathrm{Sc}$ chemical ordering and is potentially quite useful for the identification of other in-plane ordered 211 MAX phases.

Ordered divacancy formation in 2D MXene. Now that the ordered structure of the parent phase is established, we turn our attention to its MXene. Figure 1f plots the XRD patterns of $\left(\mathrm{Mo}_{2 / 3} \mathrm{Sc}_{1 / 3}\right)_{2} \mathrm{AlC}$ powders, (i) before etching in hydrofluoric acid (ii) after etching in hydrofluoric acid (HF) and (iii) with subsequent intercalation with the organic base, tetrabutylammonium hydroxide (TBAOH). The corresponding $c$ lattice parameters (LPs), expressed in hexagonal coordinates, are 13.9, 19.4 and 37.7 $\AA$. The first is typical of the 211 MAX phases, the second typical of a 211 MXene $^{18}$ and the third typical of a 211 MXene intercalated with $\mathrm{TBAOH}^{25}$. Note that the total disappearance of all but the (000l) MXene peaks implies that the entire sample was converted to MXene. The delaminated MXene will henceforth be referred to as $d-\mathrm{Mo}_{1.33} \mathrm{C}$.

After TBAOH treatment, the MXene flakes delaminate spontaneously in water. The colloidal suspension, comprising of delaminated flakes, was filtered through a nanoporous polypropylene membrane to obtain free-standing $d-\mathrm{Mo}_{1.33} \mathrm{C}$ 'paper' (Supplementary Fig. 6). XRD of this 'paper' (Fig. If (topmost scan)) shows quite a broad asymmetric (0002) peak around $8^{\circ}$, which corresponds to a $c$ - LP of $22.1 \AA$. EDX of the sample before, and after, etching (Supplementary Fig. 3) shows that both $\mathrm{Al}$ and Sc are absent after etching. Like all other MXenes, the Al layers are replaced by $\mathrm{F}$ and $\mathrm{O}$-based terminations ${ }^{16}$. X-ray photoelectron spectroscopy (XPS) measurements on the $d-\mathrm{Mo}_{1.33} \mathrm{C}$ 'paper' were performed for detailed identification and quantification of the terminating species (Supplementary 
a

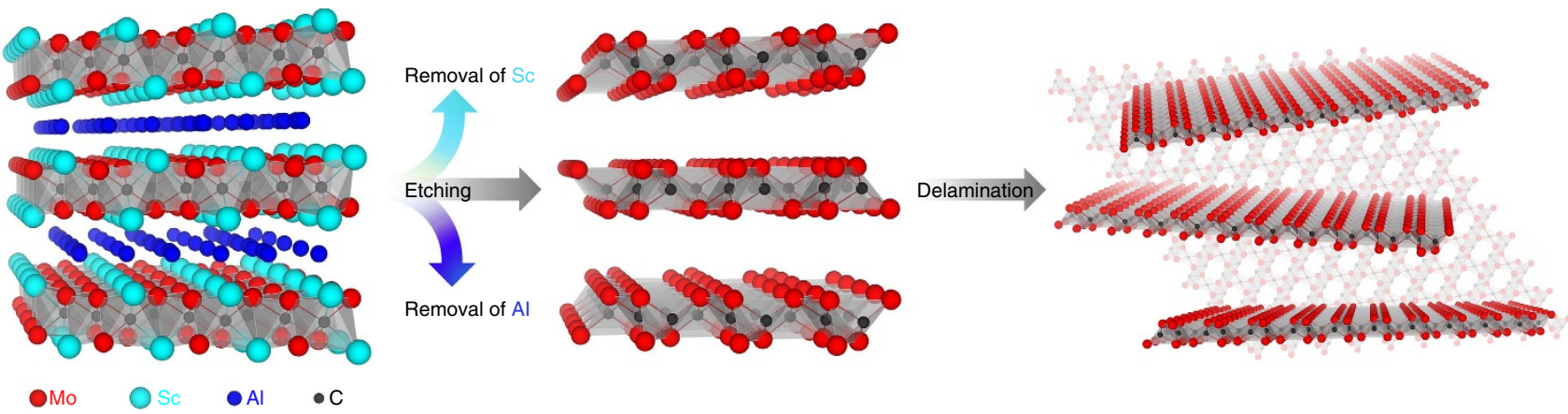

b

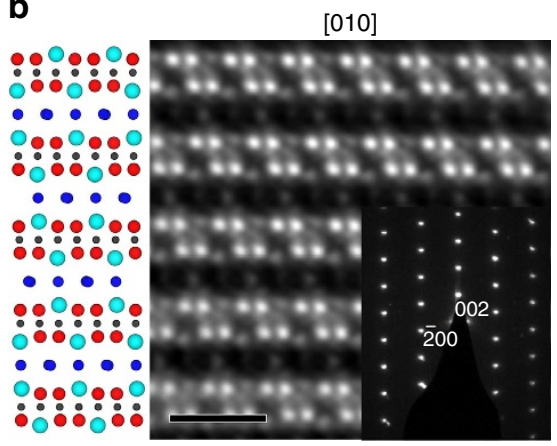

C

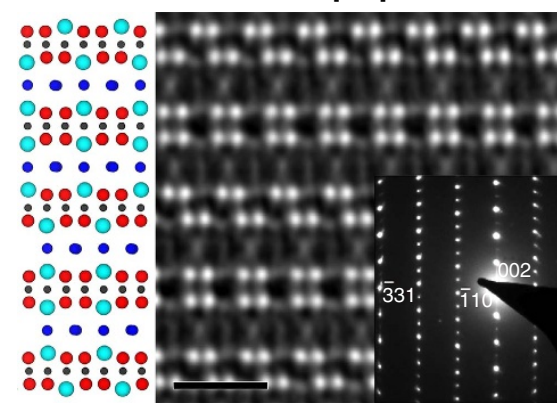

d

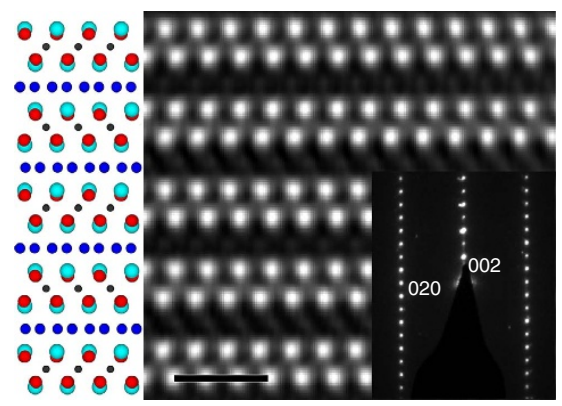

e

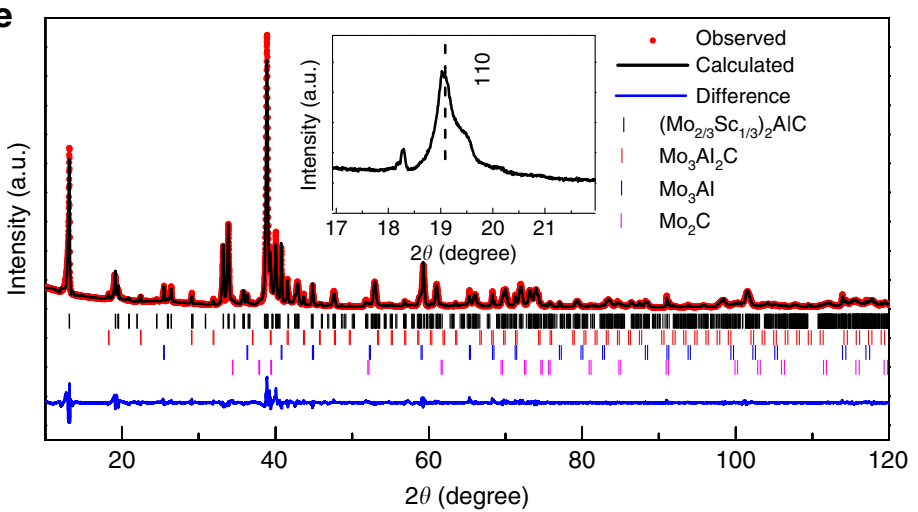

f

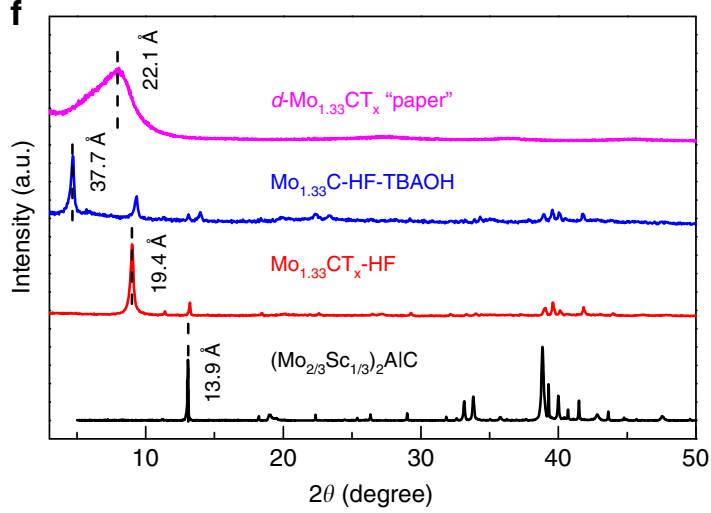

Figure 1 | In-plane chemical ordering of $\left(\mathbf{M o}_{2 / 3} \mathbf{S c}_{1 / 3}\right)_{2} \mathbf{A I C}$ leading to $\mathbf{M o}_{1.33} \mathbf{C} \mathbf{M X e n e}$ with ordered divacancies. (a) $\mathrm{Schematic}$ of $\left(\mathrm{Mo}_{2 / 3} \mathrm{SC}_{1 / 3}\right)_{2} \mathrm{AlC}$ before etching (left panel), after etching (middle panel) and after delamination (right panel). Background in the right panel shows the plane view of $\mathrm{Mo}_{1.33} \mathrm{C}$ MXene with ordered divacancies. (b-d) In-plane chemical ordering of the MAX phase evident from STEM images along the [010], [110] and [100] zone axis, respectively, with corresponding selected area electron diffraction (SAED). Schematics to the left of each image represent the corresponding atomic arrangements assuming the structure is the monoclinic space group $\mathrm{C} 2 / \mathrm{c}(15)$. (e) Rietveld refinement of XRD of sample with nominal composition ( $\mathrm{Mo}_{2} /$ $\left.{ }_{3} \mathrm{Sc}_{1 / 3}\right)_{2} \mathrm{AlC}$ assuming same space group as above. (f) XRD pattern of $\left(\mathrm{Mo}_{2 / 3} \mathrm{Sc}_{1 / 3}\right)_{2} \mathrm{AIC}$ before (black), after $\mathrm{HF}$ etching (red), and TBAOH intercalation (blue) and delamination (purple). Scale bars in (b-d) correspond to $1 \mathrm{~nm}$.

Fig. 7 and Supplementary Table 4), giving a chemical formula of $\mathrm{Mo}_{1.2} \mathrm{CO}_{0.7}(\mathrm{OH})_{0.5} \mathrm{~F}_{1.1} \cdot 0.4 \mathrm{H}_{2} \mathrm{O}_{\text {ads. }}$ (using $\mathrm{C}$ as the base) with an error of $< \pm 0.2$. Most interesting is that the material contains more $-\mathrm{F}$ terminations compared to the corresponding $d-\mathrm{Mo}_{2} \mathrm{CT}_{x}$ 'paper', for which XPS gives the chemical formula $\mathrm{Mo}_{2} \mathrm{C}_{0.9} \mathrm{O}_{1.0}(\mathrm{OH})_{1.2} \mathrm{~F}_{0.1} \cdot 0.4 \mathrm{H}_{2} \mathrm{O}_{\text {ads. }}$ (using $\mathrm{Mo}$ as the base) ${ }^{26}$. The significant difference in surface terminations between the Mo-based MXene with and without vacancies will undoubtedly influence the material properties, which is a topic of future investigations. We also note in passing that $\left(\mathrm{Mo}_{2 / 3} \mathrm{Sc}_{1 / 3}\right)_{2} \mathrm{AlC}$ can be etched by a combination of $\mathrm{LiF}$ and $\mathrm{HCl}$ (Supplementary Figs 3 and 4). Here again, after etching, the $\mathrm{Al}$ and $\mathrm{Sc}$ atoms are completely removed and replaced by $\mathrm{F}, \mathrm{O}$ and some $\mathrm{Cl}$.

An overview of the emerging monolayered structure can be seen in Fig. 2a, where a HAADF-STEM image of a single flake- with lateral dimensions $>1 \mu \mathrm{m}$-is shown covering a hole in a holey carbon TEM grid. A medium magnification image and its Fast Fourier Transform (Fig. 2b) reveal a hexagonal-based crystal with chain-like features. The latter can be better seen in Fig. 2c, which clearly reveals the individual Mo atomic positions. When this micrograph is compared, side by side and at the same scale, with the one obtained from our DFT calculations (Fig. 2d) the agreement is excellent both qualitatively and quantitatively. In both cases, the undulating Mo-atomic chains are separated by $\approx 4.7 \AA$; the projected interatomic distance is $\approx 1.9 \AA$. The presence of divacancies results in a distinctive chain-like appearance, forming a sinusoidal pattern, which is mirrored in its neighbouring chain. In these micrographs the $\mathrm{C}$ atoms exhibit negligible contrast such that only the Mo atoms are imaged. 

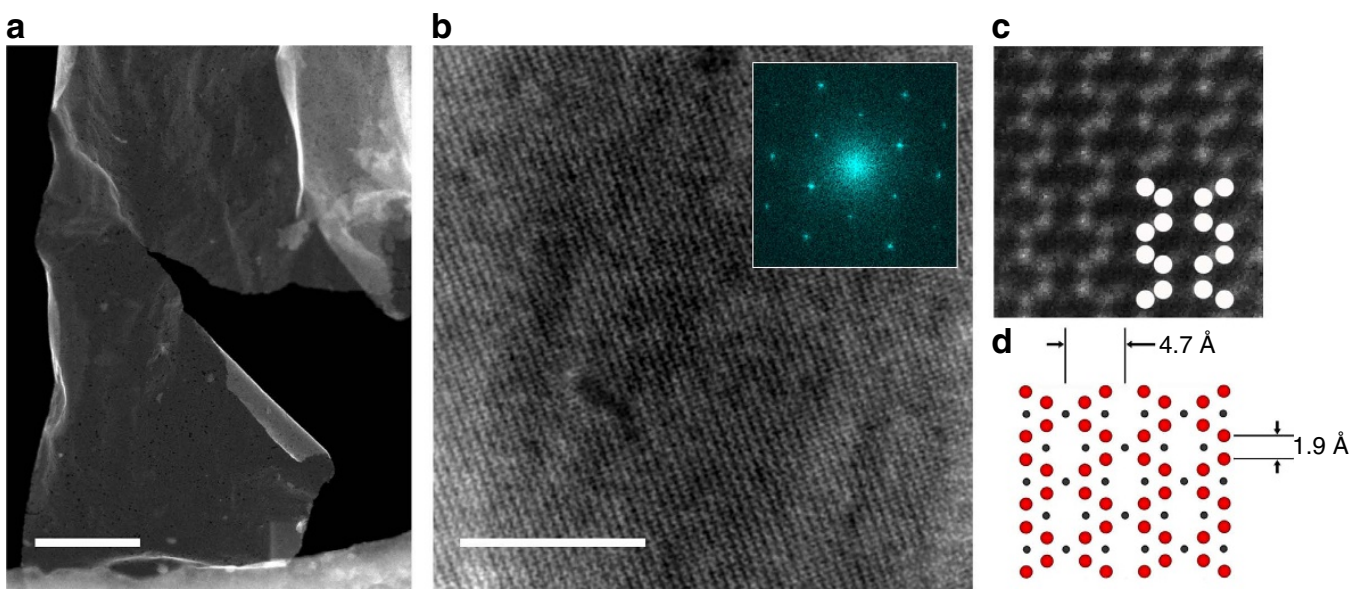

Figure 2 | Top view of HAADF-STEM of single $\mathbf{M o}_{1.33} \mathbf{C}$ sheet with ordered divacancies. (a) Low-magnification image of single flake with lateral dimensions $>1 \mu \mathrm{m}$. (b) Higher magnification, with the FFT of the original image in (a) shown in the inset. (c) Atomically resolved image with overlaid schematic atomic structure in comparison to (d) ideal atomic structure from theoretically simulated parent MAX phase. The scale in $\mathbf{c}, \mathbf{d}$ is identical. Scale bar in a corresponds $200 \mathrm{~nm}$ and scale bar in $\mathbf{b}$ corresponds to $10 \mathrm{~nm}$.

In 2015 we reported on a $\mathrm{Mo}_{2} \mathrm{C}$ MXene obtained by etching a MAX-phase-like material $\mathrm{Mo}_{2} \mathrm{Ga}_{2} \mathrm{C}$ (ref. 27). The $\mathrm{Mo}_{1.33} \mathrm{C}$ flakes synthesized here are equivalent to $\mathrm{Mo}_{2} \mathrm{C}$, albeit with ordered vacancies in the position of every third Mo atom. Consequently, $\mathrm{Mo}_{2} \mathrm{C}$ and $\mathrm{Mo}_{1.33} \mathrm{C}$ allow a detailed exploration of the effect of divacancies on properties.

Transport properties of $\mathbf{M o}_{1.33} \mathbf{C}$ 'paper'. At $33.7 \mu \Omega \mathrm{m}$ the room temperature (RT) resistivity of our $12 \mu \mathrm{m}$-thick $d-\mathrm{Mo}_{1.33} \mathrm{C}$ 'paper' is about 4 orders of magnitude lower than that reported for a $\sim 9 \mu \mathrm{m}$-thick $d-\mathrm{Mo}_{2} \mathrm{C}$ 'paper', namely $0.6 \Omega \mathrm{m}^{26}$. To the best of our knowledge, this is the highest-conductivity MXene 'paper' ever reported. Furthermore, it should be noted that no efforts were made to remove the $\mathrm{TBAOH}$ between the layers that previous work has shown increases the resistance of $\mathrm{Mo}_{2} \mathrm{C}$ films ${ }^{26}$. The presence of vacancies has been shown to influence the conductivity in other materials, for instance, in $\mathrm{TiO}_{2}$ (ref. 28). Our finding that the resistivity is so much lower in the presence of vacancies is intriguing and is currently being explored.

Electrochemical properties of $\mathrm{Mo}_{1.33} \mathrm{C}$ MXene. Previously, the capacitances of $\mathrm{Ti}_{3} \mathrm{C}_{2}, \mathrm{Mo}_{2} \mathrm{TiC}_{2}$ and $\mathrm{Mo}_{2} \mathrm{C}$-based electrodes, in $1 \mathrm{M} \mathrm{H}_{2} \mathrm{SO}_{4}$ electrolyte, were reported to be quite high ${ }^{14,24,26}$. For comparison, our $d-\mathrm{Mo}_{1.33} \mathrm{C}$ 'paper' electrodes were evaluated. When the cyclic voltammograms, $\mathrm{CV}$, obtained at scan rates in the $2-1,000 \mathrm{mV} \mathrm{s}^{-1}$ range (Fig. 3a) are compared, it is clear that, at all scan rates, several broad peaks lead to large deviations from the rectangular voltammograms expected from double-layer capacitance alone. For example, at $2 \mathrm{mV} \mathrm{s}^{-1}$, broad anodic peaks $(0.23,0.11,-0.20$, and $0.23 \mathrm{~V}$ versus $\mathrm{Ag} / \mathrm{AgCl})$ and cathodic peaks $(0.16,-0.20$, and $-0.30 \mathrm{~V}$ versus $\mathrm{Ag} / \mathrm{AgCl})$ are present. Note that the voltammetric response of $\mathrm{MoO}_{3}$ electrodes tested in $\mathrm{H}_{2} \mathrm{SO}_{4}$ and other aqueous electrolytes ${ }^{29}$ also consist of broad peaks. These common features are not surprising given that $\mathrm{Mo}_{1.33} \mathrm{C}$, like all other MXenes ${ }^{14,26}$, is terminated with $-\mathrm{O},-\mathrm{OH}$ and $-\mathrm{F}$ functional groups ${ }^{16}$.

Figure $3 \mathrm{~b}$ plots, and Supplementary Table 5 lists, the scan rate dependencies of the specific capacitances. At $2 \mathrm{mVs}^{-1}$, the volumetric capacitances-of a $3 \mu \mathrm{m}$-thick electrode-were $1,153 \mathrm{~F} \mathrm{~cm}^{-3}\left(339 \mathrm{~F} \mathrm{~g}^{-1}\right)$. These record values are, respectively, $\sim 65 \%$ and $\sim 28 \%$ higher than those reported for a $2 \mu \mathrm{m}$-thick $\mathrm{Mo}_{2} \mathrm{C}$ paper (red circles in Fig. $\left.3 \mathrm{~b}\right)^{26}$ or a $5 \mu$ m-thick $\mathrm{Ti}_{3} \mathrm{C}_{2} \mathrm{~T}_{x}$ electrode (green circles in Fig. $3 \mathrm{~b})^{14}$, respectively. At $1,000 \mathrm{mV} \mathrm{s}^{-1}$ capacitance values of $555 \mathrm{~F} \mathrm{~cm}^{-3}\left(163 \mathrm{Fg}^{-1}\right)$ were still reached. For a thicker, $12 \mu \mathrm{m}$ electrode, the capacitance values at 1,102 and $108 \mathrm{~F} \mathrm{~cm}^{-3}$ at 2 and $1,000 \mathrm{mV} \mathrm{s}^{-1}$, respectively, remain relatively high. Undoubtedly, the excellent electronic conductivity of this material and high density of 'paper' electrodes $\left(\sim 3.4 \mathrm{~g} \mathrm{~cm}^{-3}\right)$ play an important role in these record capacitance values.

The results of galvanostatically charging the half cell at a current density of $10 \mathrm{Ag}^{-1}$ (Supplementary Fig. 8) reveal slight distortion from linear voltage profiles that would be expected for double-layer capacitance alone (inset), consistent with the CVs. After 10,000 charge/discharge cycles, $100 \%$ Coulombic efficiency and $84 \%$ of the initial capacitance were retained. The latter suggests that the high number of divacancies decreases the stability of $\mathrm{Mo}_{1.33} \mathrm{C}$ slightly in sulfuric acid, as compared to $\mathrm{Mo}_{2} \mathrm{C}$ MXene films, which when tested in the same way showed no loss in capacitance ${ }^{26}$.

\section{Discussion}

To shed more light on the underlying physics, the CV data were analysed to characterize the extent of double-layer capacitive effects to the total charge stored assuming ${ }^{30}$ :

$$
I=a v^{b}
$$

where $I$ is the measured current at a given potential, $v$ is the scan rate, and $a$ and $b$ are fitting parameters obtained from $\log I$ versus $\log v$ plots (inset in Fig. $3 \mathrm{c}$ ). The resulting $b$-values (Fig. 3c) fall in the $0.84-0.97$ range. Since $b$-values near 0.5 are indicative of diffusion-limited Faradaic intercalation processes, and those close to 1.0 are indicative of capacitive currents, the values obtained here suggest that, at all but the lowest potentials of $-0.2 \mathrm{~V}$ versus $\mathrm{Ag} / \mathrm{AgCl}$, the measured current is capacitive in nature. Further quantification of the capacitive contribution to the total current was analysed using the Wang et al. ${ }^{31}$ approach. As shown in Fig. 3d, the capacitive contribution to the total current increases from $45 \%$ at $2 \mathrm{mV} \mathrm{s}^{-1}$ to $79 \%$ at $500 \mathrm{mV} \mathrm{s}^{-1}$. The high $b$-values, broad peaks in the CVs, and high specific capacitances observed suggest that pseudocapacitance is the major operative mechanism herein as postulated for other MXenes ${ }^{14,24,32,33}$. In the case of $\mathrm{Ti}_{3} \mathrm{C}_{2}$, direct evidence exists in terms of in situ X-ray absorption spectroscopy ${ }^{32}$. What is unclear at this time is whether the divacancies enhanced the capacitance 

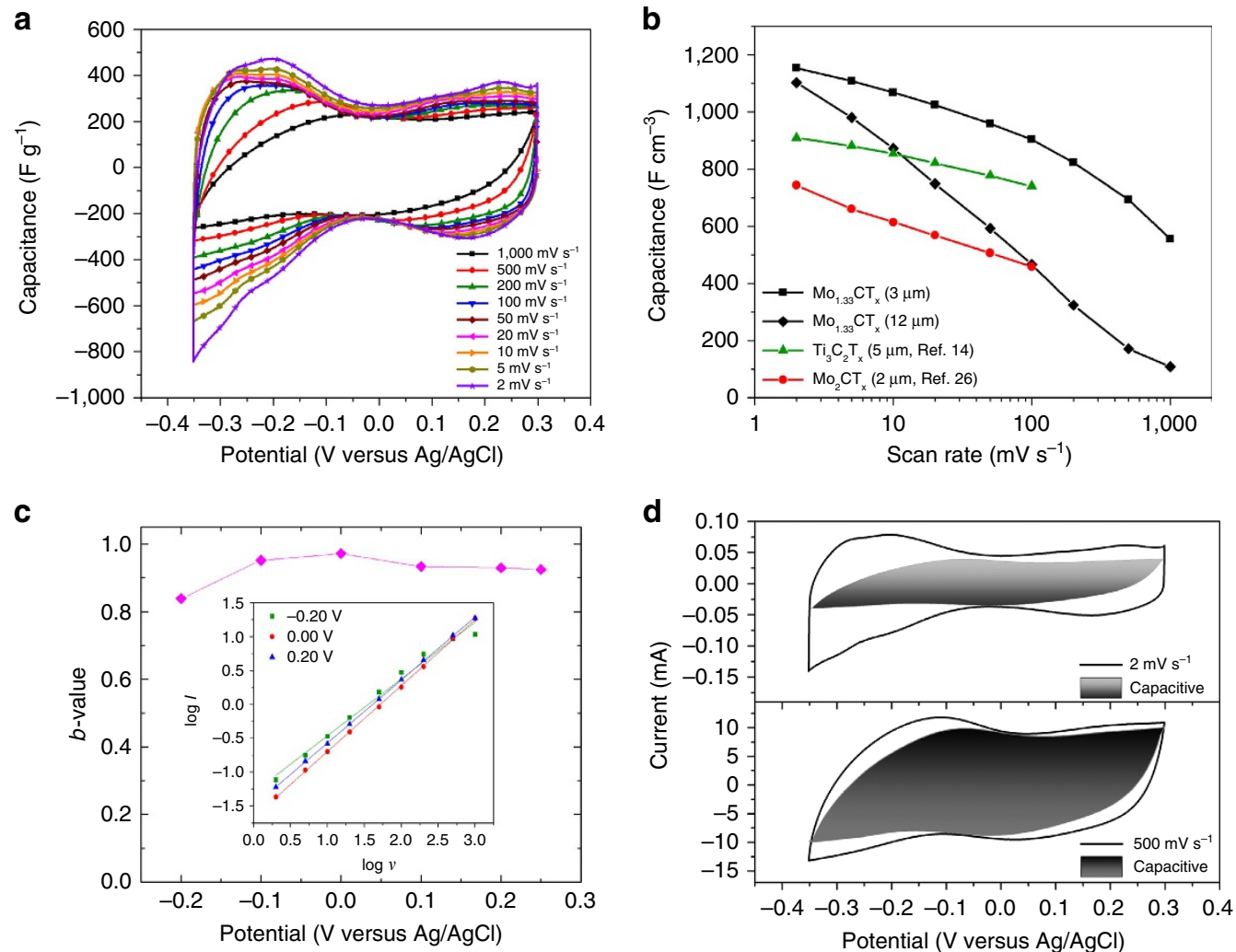

Figure 3 | Electrochemical performance of $\mathbf{M o}_{1.33} \mathbf{C}$ in $\mathbf{1} \mathbf{M} \mathbf{H}_{\mathbf{2}} \mathbf{S O}_{\mathbf{4}}$. Experiments were conducted in a three-electrode Swagelok cell. (a) Cyclic voltammograms of the $3 \mu \mathrm{m}$-thick electrode. (b) Scan rate dependence of specific capacitance of 3 and $12 \mu \mathrm{m}$ thick free-standing electrodes. Also plotted are previous results on $\mathrm{Ti}_{3} \mathrm{C}_{2} \mathrm{~T}_{x}$ clay ${ }^{14}$ and $\mathrm{Mo}_{2} \mathrm{CT}_{x}{ }^{26}$ (c) $b$-values for a $3 \mu \mathrm{m}$-thick film. Inset plots $\log /$ versus log $v$ (see equation (1) in text). (d) $\mathrm{CV}$ partition analysis showing capacitive contribution to total current at select scan rates.

values by increasing the electrochemically active sites and/or by increasing the fraction of the electrode accessible to the electrolyte. Clearly, more work, some of which is ongoing and beyond the scope of this paper, is needed to answer some of these fundamental questions.

To summarize, by selectively extracting both the Sc and $\mathrm{Al}$ atoms from a chemically in-plane ordered quaternary MAX phase $\left(\mathrm{Mo}_{2 / 3} \mathrm{Sc}_{1 / 3}\right)_{2} \mathrm{AlC}$, we realized a $2 \mathrm{D} \mathrm{Mo}_{1.33} \mathrm{C}$ MXene with ordered divacancies. The latter, in turn, leads to higher conductivities and record volumetric capacitances for pure MXene films. It follows that an alternative path for propertytuning of $2 \mathrm{D}$ materials now exists. The implications and ramifications of this work are as follows: our 2D flakes with ordered vacancies can be readily made in aqueous environments, using a top-down approach, with yields that are close to $100 \%$. The fact that the final flakes are less dense than their counterparts without vacancies is also noteworthy when specific properties are sought. We note in passing that the Sc used can easily be recycled. Based on the results shown herein, it is reasonable to believe that this approach may be of importance for energy storage and other applications.

\section{Methods}

Theoretical calculations. Electronic structure calculations were carried out using DFT using the projector augmented wave method ${ }^{34,35}$ with the Perdew-Burke-Ernzerhof (PBE) ${ }^{36}$ non-spin polarized generalized gradient approximation (GGA) exchange-correlation potential as implemented within the Vienna ab-initio simulation package (VASP) ${ }^{37-39}$. The electronic wave functions were expanded in a plane-wave basis set with a cutoff energy of $400 \mathrm{eV}$, and for sampling of the Brillouin zone we used Monkhorst-Pack $k$-point meshes to achieve a total energy convergence of less than $0.5 \mathrm{eV}$ per atom ${ }^{40}$. Calculations are performed at zero temperature and pressure, and all structures were fully relaxed.
To determine the dynamical stability of the studied ordered structure, we performed phonon calculations using finite displacement method along with Phonopy ${ }^{41}$.

Synthesis of $\left(\mathbf{M o}_{\mathbf{2} / \mathbf{3}} \mathbf{S c}_{\mathbf{1 / 3}}\right)_{\mathbf{2}} \mathbf{A I C} \mathbf{M A X}$ phase. The powders used herein were graphite (99.999\%), Mo (99.99\%), (Sigma-Aldrich), Al (99.8\% Alfa Aesar), and $\mathrm{Sc}$ (99.99\% Stanford Advanced Material). To obtain the $\left(\mathrm{Mo}_{2 / 3} \mathrm{Sc}_{1 / 3}\right)_{2} \mathrm{AlC}$ powder samples, a stoichiometric amount was mixed in an agate mortar, heated to $1,500{ }^{\circ} \mathrm{C}$ in an alumina crucible under flowing argon and held at that temperature for $20 \mathrm{~h}$. After cooling down to RT in the furnace, a loosely packed powder was obtained. The powder was crushed and sieved through a 450 mesh sieve (particle size $<32 \mu \mathrm{m}$ ). This fine powder was then used for XRD, EDX, STEM and MXene preparation.

Synthesis of $\mathbf{M o}_{1.33} \mathbf{C} \mathbf{M X e n e}$. One gram of the sieved powder was added to $20 \mathrm{ml} 48 \% \mathrm{HF}$, stirring for $24 \mathrm{~h}$ at RT. After etching, the suspension was filtered, dispersed in water, and filtrated again. The washing procedure was repeated 5 times to remove any remaining acid and/or reaction products. The powder on the filtration membrane was dried at RT for $12 \mathrm{~h}$. The dried powder was kept for further experiments and characterization. For exfoliation, $0.1 \mathrm{~g}$ of the etched powder and $1 \mathrm{ml}$ TBAOH were added to a centrifuge tube, which was shaken manually for $5 \mathrm{~min}$. The tube was then centrifuged at 6,000 r.p.m. for $5 \mathrm{~min}$ to remove the supernatant. Water was added to the tube to wash away the remaining $\mathrm{TBAOH}$, after which the water was poured out. The process was repeated three times. The water was added carefully not to agitate the powder, in order not to delaminate the MXene. Finally, water was added to the powder and shaken for 5 min for delamination into single- or few- layered MXene. Another synthesis approach is to use $\mathrm{LiF}$ and $\mathrm{HCl}$. Two gram of $\mathrm{LiF}$ was dissolved in $30 \mathrm{ml} 12 \mathrm{M} \mathrm{HCl}$. One gram of the sieved MAX powder was added to the $\mathrm{LiF} / \mathrm{HCl}$ solution, stirring for $48 \mathrm{~h}$ at $35^{\circ} \mathrm{C}$. After etching, the suspension was filtered. Powder was added to a centrifuge tube and washed three times with $1 \mathrm{M} \mathrm{HCl}$ to remove excess $\mathrm{LiF}$, and then three times with water. The $\mathrm{HCl}$-washed slurry was thereafter washed three times with $1 \mathrm{M} \mathrm{LiCl}$, and then three times with water. The supernatant was removed by centrifuging at 3,000 r.p.m. for 5 min using the above $\mathrm{HCl}$ and $\mathrm{LiCl}$ washing procedures. Finally, water was added to the washed slurry, and shaken for $5 \mathrm{~min}$ for delamination into single, or few, layered MXene. 
Materials characterization. XRD scans of the $\left(\mathrm{Mo}_{2 / 3} \mathrm{Sc}_{1 / 3}\right)_{2} \mathrm{AlC}$ powders and $d-\mathrm{Mo}_{1.33} \mathrm{C}$ 'paper' were carried out on a diffractometer (Panalytical X'pert). The XRD diffractogram of the $\left(\mathrm{Mo}_{2 / 3} \mathrm{Sc}_{1 / 3}\right)_{2} \mathrm{AlC}$ powders was analysed by the Rietveld refinement method, using the FULLPROF code ${ }^{42,43}$. Refined parameters were: background parameters, scale factors, $\mathrm{X}$ and $\mathrm{Y}$ profile parameters for peak width, Lattice parameters, and atomic positions for all phases. The occupancies of $\mathrm{Mo} / \mathrm{Sc}$ were refined manually by stepwise varying the ratio of $\mathrm{Mo} / \mathrm{Sc}$.

For the in-plane ordered MAX and MXene analyses, STEM combined with high angle-annular dark-field imaging (STEM-HAADF) and EDX analysis with a super$\mathrm{X}$ EDX detector was performed in the double-corrected Linköping FEI Titan ${ }^{3}$ $60-300$, operated at 300 and $60 \mathrm{kV}$, respectively. Selective area electron diffraction was performed on a FEI Tecnai T20 transmission electron microscope operated at $200 \mathrm{kV}$. Delaminated flakes were dispersed onto standard holey amorphous carbon support films suspended by Cu grids (SPI Supplies). Atomic resolution STEM-HAADF images were recorded using an optimized $30 \mathrm{mrad}$ convergence angle, which provided $\AA$-resolution probes at $60 \mathrm{kV}$, and $50 \mathrm{pA}$ probe current. The HAADF detector's inner acceptance angle was set to $25 \mathrm{mrad}$.

XPS measurements were performed on a free-standing $d-\mathrm{Mo}_{1.33} \mathrm{CT}_{x}$ 'paper' using monochromatic $\mathrm{Al}-\mathrm{K}_{\alpha}(1,486.6 \mathrm{eV})$ radiation in a Kratos AXIS Ultra ${ }^{D L D}$ system. The sample was mounted on a double-sided tape and grounded to the sample stage with copper contacts. Charge neutralization was performed using a co-axial, low-energy $(\sim 0.1 \mathrm{eV})$ electron flood source to avoid shifts in the recorded binding energy, BE. XPS spectra were recorded for F 1s, O 1s, C 1s, Al 2p, and Mo $3 \mathrm{~d}$. The analyser pass energy used for all the regions was $20 \mathrm{eV}$ with a step size of $0.1 \mathrm{eV}$. The BE scale of all XPS spectra was referenced to the Fermi edge, which was set to a $\mathrm{BE}$ of $0 \mathrm{eV}$. The peak fitting was carried out using CasaXPS Version 2.3.16 RP 1.6 as in ref. 16.

Preparation of $\boldsymbol{d}-\mathbf{M o}_{1.33} \mathbf{C}$ 'paper'. The $\mathrm{Mo}_{1.33} \mathrm{C}$ 'paper' was prepared from $d-\mathrm{Mo}_{1.33} \mathrm{C}$ flakes obtained by HF etching of $\left(\mathrm{Mo}_{2 / 3} \mathrm{Sc}_{1 / 3}\right)_{2} \mathrm{AlC}$, followed by TBAOH intercalation as described above. After TBOAH treatment, the sediment was mixed with DI water $\left(1 \mathrm{~g} \mathrm{Mo}_{1.33} \mathrm{C}\right.$ per $10 \mathrm{ml}$ of water) and de-aerated using $\mathrm{Ar}$ gas, followed by sonication in an ice-cooled ultrasonic bath for $1 \mathrm{~h}$. The mixture was then centrifuged for $1 \mathrm{~h}$ at 5,000 r.p.m., and the supernatant was collected. The latter was vacuum-filtered onto nanoporous polypropylene membranes (Celgard $3501,0.064 \mu \mathrm{m}$ pore size, Celgard LLC) in air. The $d-\mathrm{Mo}_{1.33} \mathrm{C}$ 'paper' was easily peeled off from the membrane to obtain free-standing $\approx 12 \mu \mathrm{m}$ and $\approx 3 \mu \mathrm{m}$ thick 'paper'.

Transport measurements. A linear, four-point probe geometry was used to measure the RT resistivity of $d$ - $\mathrm{Mo}_{1.33} \mathrm{C}$ 'paper' using a Physical Property Measurement System (PPMS) (Quantum Design, San Diego). Gold wires were attached to the films using silver paint. The errors in the resistivity values are estimated to be $\sim 5 \%$, corresponding to the uncertainty in sample thicknesses.

Electrochemical testing. $\mathrm{Mo}_{1.33} \mathrm{C}$ 'paper', $\approx 3 \mu \mathrm{m}$ and $\approx 12 \mu \mathrm{m}$ thick films were used without further preparation as working electrodes in a three-electrode configuration. YP-50 activated carbon (Kuraray, Japan) electrodes were prepared and used as overcapacitive counter electrodes, according to the procedures outlined in ref. 14: A stainless steel Swagelok half-cell was assembled with $\mathrm{Mo}_{1.33} \mathrm{C}$ 'paper' on a gold foil current collector as the working electrode, overcapacitive activated carbon on a Pt foil current collector as the counter electrode and $\mathrm{Ag} / \mathrm{AgCl}$ in $1 \mathrm{M}$ $\mathrm{KCl}$ as the reference electrode. Electrochemical tests were conducted using a potentiostat/galvanostat (Bio-logic VMP3). CVs were obtained at scan rates in the 2 to $1,000 \mathrm{mV} \mathrm{s}^{-1}$ range after an initial pre-cycling of the cell 100 times at $20 \mathrm{mV} \mathrm{s}^{-1}$. Based on our experience, pre-cycling generally helps to saturate the interlayer space between the densely packed MXene sheets with electrolyte. The same half-cells were also galvanostatically charged and discharged for 10,000 cycles at a current density of $10 \mathrm{Ag}^{-1}$. The gravimetric capacitance values, $C_{\mathrm{m}}$, were calculated from cyclic voltammetry data assuming:

$$
C_{\mathrm{m}}=\frac{1}{m \Delta E} \int_{E_{1}}^{E_{2}} \frac{I}{v} \mathrm{~d} E
$$

where $m$ is the working electrode's mass, $\Delta E$ is the potential window, $E_{1}$ and $E_{2}$ are the potential limits, $I$ is the measured current, and $v$ is the scan rate. Volumetric capacitance values were computed by multiplying the gravimetric capacitance by the working electrode density, which was $3.4 \mathrm{~g} \mathrm{~cm}^{-3}$.

Data availability. All relevant data are available from the authors upon request.

\section{References}

1. Neto, A. C., Guinea, F., Peres, N. M., Novoselov, K. S. \& Geim, A. K. The electronic properties of graphene. Rev. Mod. Phys. 81, 109 (2009).

2. Butler, S. Z. et al. Progress, challenges, and opportunities in two-dimensional materials beyond graphene. ACS Nano 7, 2898-2926 (2013).

3. Novoselov, K. S., Mishchenko, A., Carvalho, A. \& Neto, A. C. 2D materials and van der Waals heterostructures. Science 353, aac9439 (2016).
4. Robertson, A. W. et al. Spatial control of defect creation in graphene at the nanoscale. Nat. Commun. 3, 1144 (2012).

5. Nair, R. R. et al. Spin-half paramagnetism in graphene induced by point defects. Nat. Phys. 8, 199-202 (2012).

6. Liu, Y., Xiao, H. \& Goddard, W. A. Two-dimensional halide perovskites: tuning electronic activities of defects. Nano Lett. 16, 3335-3340 (2016).

7. Li, H. et al. Activating and optimizing $\mathrm{MoS}_{2}$ basal planes for hydrogen evolution through the formation of strained sulphur vacancies. Nat. Mater. 15, $48-53$ (2016).

8. Amani, M. et al. Near-unity photoluminescence quantum yield in $\mathrm{MoS}_{2}$. Science 350, 1065-1068 (2015).

9. Yang, X., Cheng, C., Wang, Y., Qiu, L. \& Li, D. Liquid-mediated dense integration of graphene materials for compact capacitive energy storage. Science 341, 534-537 (2013).

10. El-Kady, M. F., Strong, V., Dubin, S. \& Kaner, R. B. Laser scribing of high-performance and flexible graphene-based electrochemical capacitors. Science 335, 1326-1330 (2012).

11. Tao, Y. et al. Towards ultrahigh volumetric capacitance: graphene derived highly dense but porous carbons for supercapacitors. Sci. Rep. 3, 2975 (2013).

12. Brezesinski, T., Wang, J. S., Tolbert, H. \& Dunn, B. Ordered mesoporous $\alpha-\mathrm{MoO}_{3}$ with iso-oriented nanocrystalline walls for thin-film pseudocapacitors. Nat. Mater. 9, 146-151 (2010).

13. Hu, C. C., Chang, K. H., Lin, M. C. \& Wu, Y. T. Design and tailoring of the nanotubular arrayed architecture of hydrous $\mathrm{RuO}_{2}$ for next generation supercapacitors. Nano Lett. 6, 2690-2695 (2006).

14. Ghidiu, M., Lukatskaya, M. R., Zhao, M. Q., Gogotsi, Y. \& Barsoum, M. W. Conductive two-dimensional titanium carbide 'clay' with high volumetric capacitance. Nature 516, 78-81 (2014).

15. Naguib, M. et al. Two-dimensional nanocrystals produced by exfoliation of $\mathrm{Ti}_{3} \mathrm{AlC}_{2}$. Adv. Mater. 23, 4248-4253 (2011).

16. Halim, J. et al. X-ray photoelectron spectroscopy of select multi-layered transition metal carbides (MXenes). App. Surf. Sci. 362, 406-417 (2016).

17. Lukatskaya, M. R. et al. Cation intercalation and high volumetric capacitance of two-dimensional titanium carbide. Science 341, 1502-1505 (2013).

18. Naguib, M. et al. New two-dimensional niobium and vanadium carbides as promising materials for Li-ion batteries. J. Am. Chem. Soc. 135, 15966-15969 (2013).

19. Peng, Q. et al. Unique lead adsorption behavior of activated hydroxyl group in two-dimensional titanium carbide. J. Am. Chem. Soc. 136, 4113-4116 (2014).

20. Dillon, A. D. et al. Highly-conductive, optical-quality films from solutionprocessed 2D titanium carbide (MXene). Adv. Funct. Mater. 26, 4162-4168 (2016).

21. Shahzad, F. et al. Electromagnetic interference shielding with 2D transition metal carbides (MXenes). Science 353, 1137-1140 (2016).

22. Liu, Z. et al. $\left(\mathrm{Cr}_{2 / 3} \mathrm{Ti}_{1 / 3}\right)_{3} \mathrm{AlC}_{2}$ and $\left(\mathrm{Cr}_{5 / 8} \mathrm{Ti}_{3 / 8}\right)_{4} \mathrm{AlC}_{3}$ : New MAX-phase Compounds in Ti-Cr-Al-C System. J. Am. Cer. Soc 97, 67-69 (2014).

23. Meshkian, R. et al. Theoretical stability and materials synthesis of a chemically ordered MAX phase, $\mathrm{Mo}_{2} \mathrm{ScAlC}_{2}$, and its two-dimensional derivate $\mathrm{Mo}_{2} \mathrm{ScC}_{2}$ MXene. Acta. Mater 125, 476-480 (2017).

24. Anasori, B. et al. Two-dimensional, ordered, double transition metals carbides (MXenes). ACS Nano 9, 9507-9516 (2015).

25. Naguib, M., Unocic, R. R., Armstrong, B. L. \& Nanda, J. Large-scale delamination of multi-layers transition metal carbides and carbonitrides 'MXenes'. Dalton Trans. 44, 9353-9358 (2015).

26. Halim, J. et al. Synthesis and characterization of 2D molybdenum carbide (MXene). Adv. Func. Mater. 26, 3118-3127 (2016).

27. Meshkian, R. et al. Synthesis of two-dimensional molybdenum carbide, $\mathrm{Mo}_{2} \mathrm{C}$, from the gallium based atomic laminate $\mathrm{Mo}_{2} \mathrm{Ga}_{2}$ C. Scr. Mater. 108, 147-150 (2015).

28. Kwon, D. et al. Atomic structure of conducting nanofilaments in $\mathrm{TiO}_{2}$ resistive switching memory. Nat. Nanotechnol. 5, 148-153 (2010).

29. Mendoza-Sánchez, B., Brousse, B. T., Ramirez-Castro, C., Nicolosi, V. \& Grant, P. S. An investigation of nanostructured thin film $\alpha-\mathrm{MoO}_{3}$ based supercapacitor electrodes in an aqueous electrolyte. Electrochim. Acta 91, 253-260 (2013).

30. Lindström, $\mathrm{H}$. et al. $\mathrm{Li}^{+}$ion insertion in $\mathrm{TiO}_{2}$ (Anatase). 1 chronoamperometry on CVD films and nanoporous films. J. Phys. Chem. B 101, 7710-7716 (1997).

31. Wang, J., Polleux, J., Lim, J. \& Dunn, B. Pseudocapacitive contributions to electrochemical energy storage in $\mathrm{TiO}_{2}$ (Anatase) nanoparticles. J. Phys. Chem. C 111, 14925-14931 (2004).

32. Lukatskaya, M. R. et al. Probing the mechanism of high capacitance in $2 \mathrm{D}$ titanium carbide using in situ X-ray absorption spectroscopy. Adv. Energy Mater 5, 1500589 (2015).

33. Levi, D. M. et al. Solving the capacitive paradox of 2D MXene using electrochemical Quartz-crystal admittance and in situ electronic conductance measurements. Adv. Energy Mater 5, 1400815 (2015). 
34. Blöchl, P. E. Projector augmented-wave method. Phys. Rev. B 50, 17953-17979 (1994).

35. Kresse, G. \& Joubert, D. From ultrasoft pseudopotentials to the projector augmented-wave method. Phys. Rev. B 59, 1758-1775 (1999).

36. Perdew, J. P., Burke, K. \& Ernzerhof, M. Generalized gradient approximation made simple. Phys. Rev. Lett. 77, 3865-3868 (1996).

37. Kresse, G. \& Hafner, J. Ab initio molecular dynamics for liquid metals. Phys. Rev. B 47, 558-561 (1993).

38. Kresse, G. \& Furthmüller, J. Efficiency of $a b$-initio total energy calculations for metals and semiconductors using a plane-wave basis set. Comput. Mater. Sci. 6, 15-50 (1996).

39. Kresse, G. \& Furthmüller, J. Efficient iterative schemes for ab initio totalenergy calculations using a plane-wave basis set. Phys. Rev. B 54, 11169-11186 (1996).

40. Monkhorst, H. J. \& Pack, J. D. Special points for Brillouin-zone integrations. Phys. Rev. B 13, 5188-5192 (1976).

41. Togo, A., Oba, F. \& Tanaka, I. First-principles calculations of the ferroelastic transition between rutile-type and $\mathrm{CaCl}_{2}$ type $\mathrm{SiO}_{2}$ at high pressures. Phys. Rev. B 78, 134106 (2008).

42. Rietveld, H. A profile refinement method for nuclear and magnetic structures. J. Appl. Crystallogr. 2, 6571 (1969).

43. Rodríguez-Carvajal, J. Recent advances in magnetic structure determination by neutron powder diffraction. Phys. B: Condens. Matter 192, 55-69 (1993).

\section{Acknowledgements}

J.R., P.O.Å.P. and M.W.B. acknowledge support from the Swedish Foundation for Strategic Research (SSF) through the Synergy Grant FUNCASE. J.R., P.O.A.P. and L.H. also acknowledge support from the Knut and Alice Wallenberg (KAW) Foundation for a Scholar Grant, a Fellowship Grant, Project funding (KAW 2015.0043), and for support to the Linköping Electron Microscopy Laboratory. The Swedish Research council is gratefully acknowledged through Projects 621-2012-4359, 622-2008-405, 621-2012-4425 and 642-2013-8020. The calculations were carried out using supercomputer resources provided by the Swedish National Infrastructure for Computing (SNIC) at the National Supercomputer Centre (NSC) and the PDC Center for High Performance Computing. We also thank Drs. S. May, E. Ju and Y. Gogotsi, all from Drexel University, for the transport measurements and the use of the electrochemical equipment, respectively.

\section{Author contributions}

J.R. initiated and supervised the study. Q.T. and R.M. conducted and analysed the synthesis, XRD and EDX. L.H. oversaw the MAX phase STEM analysis performed by J.L. The MXene STEM analysis was conducted by J.P. under supervision of P.O.A.P. M.D. performed the $a b$ initio calculations. Q.T., R.M. and J.H. conducted the Rietveld refinement, and the MXene preparation. J.H. conducted the transport and XPS measurements. S.K. conducted the capacitance tests and analysis supervised by M.W.B. Q.T., M.W.B., P.O.Å.P. and J.R. wrote the manuscript with contributions from the other coauthors. All coauthors read and commented on successive drafts of the manuscript.

\section{Additional information}

Supplementary Information accompanies this paper at http://www.nature.com/ naturecommunications

Competing interests: The authors declare no competing financial interests.

Reprints and permission information is available online at http://npg.nature.com/ reprintsandpermissions/

How to cite this article: Tao, Q. et al. Two-dimensional $\mathrm{Mo}_{1.33} \mathrm{C}$ MXene with divacancy ordering prepared from parent 3D laminate with in-plane chemical ordering. Nat. Commun. 8, 14949 doi: 10.1038/ncomms14949 (2017).

Publisher's note: Springer Nature remains neutral with regard to jurisdictional claims in published maps and institutional affiliations.

\section{(c) (i)}

This work is licensed under a Creative Commons Attribution 4.0 International License. The images or other third party material in this article are included in the article's Creative Commons license, unless indicated otherwise in the credit line; if the material is not included under the Creative Commons license, users will need to obtain permission from the license holder to reproduce the material. To view a copy of this license, visit http://creativecommons.org/licenses/by/4.0/

(C) The Author(s) 2017 